\title{
Envisioning Ubiquitous Computing
}

\author{
Stuart Reeves \\ Horizon Digital Economy Research \\ University of Nottingham, UK \\ stuart@tropic.org.uk
}

\begin{abstract}
Visions of the future are a common feature of discourse within ubiquitous computing and, more broadly, HCI. 'Envisioning', a characteristic future-oriented technique for design thinking, often features as significant part of our research processes in the field. This paper compares, contrasts and critiques the varied ways in which envisionings have been used within ubiquitous computing and traces their relationships to other, different envisionings, such as those of virtual reality. In unpacking envisioning, it argues primarily that envisioning should be foregrounded as a significant concern and interest within HCI. Foregrounding envisioning's frequent mix of fiction, forecasting and extrapolation, the paper recommends changes in the way we read, interpret and use envisionings through taking into account issues such as context and intended audience.
\end{abstract}

\section{Author Keywords}

Ubiquitous computing; vision; design fiction; forecasting; futures; scenarios; teleology.

\section{ACM Classification Keywords}

H.1.0 [Information systems]: General.

\section{General Terms}

Human Factors; Theory; Design.

\section{INTRODUCTION}

In this paper I will use the term 'envisioning' to refer to a broadly future-oriented aspect of technology design which mixes fictions, forecasts, extrapolations or projections into societal visions for technological progress. Envisioning is a widespread feature of much technological development; visions of the future have often found a place in large commercial organisations who play a significant role in research communities (such as ubicomp, HCI, etc.); for instance, BT [30], Intel [20], and Philips [24]. Some of the most recent and significant instances of envisioning within HCI have taken place in ubiquitous computing, which as others have remarked, "occupies spaces that hinge on a future yet to happen, or futures that may not ever happen" [16]. As part of this I must address the context in which envisionings are created and communicated, and, crucially for HCI, the way in which envisionings are subsequently interpreted and used by others. It is this aspect-

Permission to make digital or hard copies of all or part of this work for personal or classroom use is granted without fee provided that copies are not made or distributed for profit or commercial advantage and that copies bear this notice and the full citation on the first page. To copy otherwise, or republish, to post on servers or to redistribute to lists, requires prior specific permission and/or a fee.

CHI'12, May 5-10, 2012, Austin, Texas, USA.

Copyright 2012 ACM 978-1-4503-1015-4/12/05 ..\$10.00. specifically foregrounding how envisionings can be unpacked and 'read' - which is the main focus of this paper.

There are several characteristic interests of envisioning that make its work distinctive. It involves imagining (via various means, such as forecasts or extrapolations) what future societal situations may be. This is coupled with a concern for how those projected societal situations may (or may not) be amenable to technological intervention. Technology design is then informed by what fits with this imagined range of future societal situations. Envisioning also involves projection into the future regarding implications of existing technology trends. Finally, envisionings have an ambiguous status in that they are often entangled with fictional representations, and as such it can be difficult to separate where forecasts and extrapolations end, and fictions begin.

In addition to this particular set of orientations, envisioning typically involves one or more of a collection of methods through which the envisionings are communicated to others. These methods vary greatly, and this paper presents several of them, including future scenarios described in papers and books, promotional research videos, research 'vision statements' and proposals, statements of justification in research papers, and of course the construction and deployment of technological artefacts tied to envisionings. Envisionings are created for a range of purposes (often satisfying several at the same time), although characteristically these purposes are often implicit, rather than explicitly built into the envisioning. These purposes might be: to establish and delineate a new area of research; to justify a interest in a particular kind of technology; to inspire; to provide an accountable 'plan' for the future for funders; or to attract funding to carry out particular kinds of work. In turn, these envisionings are interpreted and deployed by others to do the work of any number of the above purposes.

I argue that these aspects of envisioning are not entirely foregrounded within technology research communities. Rather, they often come to implicitly frame discourse within the field, and configure a set of assumptions that orient ways in which our work is done. Although research on discourses of scientific and technological futures has been established within Science and Technology Studies for some time (e.g., $[9,36,21])$, this topic has had little discussion in HCI. Thus, the purpose of this paper is to foreground these implicit assumptions, and identify both the values and the dangers of envisioning. In this sense, I want to explore how envisionings can potentially be 'considered harmful' as well as 'considered useful'. 
The rest of this paper is divided into four sections. The first section surveys ubicomp envisionings, both original and more recent iterations. The second section situates these in a broader context by examining examples of historical technological envisionings, with particular focus on one of the key motivators of ubicomp - virtual reality (VR). Developing this, the third section then discusses the importance of how we interpret envisionings, exploring some of the different ways this may be done. The final section then discusses the implications of these issues for envisioning and ubiquitous computing, outlines some pros and cons of envisioning, and examines some key topics which either open new discussions in HCI or need greater prominence.

\section{ENVISIONING AND UBIQUITOUS COMPUTING ${ }^{1}$}

As Bell \& Dourish suggest, "ubiquitous computing [...] research is characterized primarily by a concern with potential future computational worlds" [4]. Early ubicomp envisionings have played an instrumental role orienting research (and popular media) understandings to a particular set of assumptions about - and a particular approach toubicomp technology design. This section locates and exhibits some of the historical ways in which ubiquitous computing has used envisioning. In doing so we must 'rehearse' an oft-repeated narrative of ubicomp's envisioning; through this I will unpack its various characteristics, including uses of forecasting, extrapolation and fiction.

\section{Ubiquitous computing's early envisioning}

In the late 80s and early 90s, researchers at Xerox PARC's Computer Sciences Lab (CSL) began envisioning a different form of computing, one that was in part influenced by disenchantment with the emerging interest in virtual reality technologies at the time. PARC researchers positioned what they called 'ubiquitous computing' technologies in direct opposition with the values of virtual reality "which attempts to make a world inside the computer" [43]. At the time this was a radical shift, borne in the context of an ascendant VR and a present environment in which the prevailing domain of computer technology was the workplace. In his 1991 paper for Scientific American (SciAm), entitled "The computer for the twenty-first century", Mark Weiser presented what has been appropriated as an envisioning for ubiquitous computing:

The most profound technologies are those that disappear. They weave themselves into the fabric of everyday life until they are indistinguishable from it. My colleagues and I at PARC think that the idea of a "personal" computer itself is misplaced, and that the vision of laptop machines, dynabooks and "knowledge navigators" is only a transitional step toward achieving the real potential of information technology. Such machines cannot truly make computing an integral, invisible part of the way people live their lives. Therefore we are trying to conceive a new way of thinking about computers in the world, one that

\footnotetext{
${ }^{1}$ For the purposes of this paper, 'ubiquitous computing' is used as a gloss for a variety of interlinked labels, including ubicomp, ambient intelligence, pervasive computing, tangible computing.
}

takes into account the natural human environment and allows the computers themselves to vanish into the background. [43]

The first aspect to note is that this paper has become, via heavy citation, the canonical reference for the ubiquitous computing envisioning. Ignoring the context of publication (i.e., in a popular science magazine), it is easy to see Weiser's description as a forecast (particularly given the title [4]), in which current technology is seen as only "transitional" and instead lays out a direction "toward achieving the real potential of information technology". Envisionings are often formed through a dissatisfaction with the limitations of existing technologies. Thus technology here is assigned a transformative power and trajectory, and, crucially, ubicomp is framed in the context of a societal mission and change (a perspective which may be traced to PARC's more recent work, e.g., [5]).

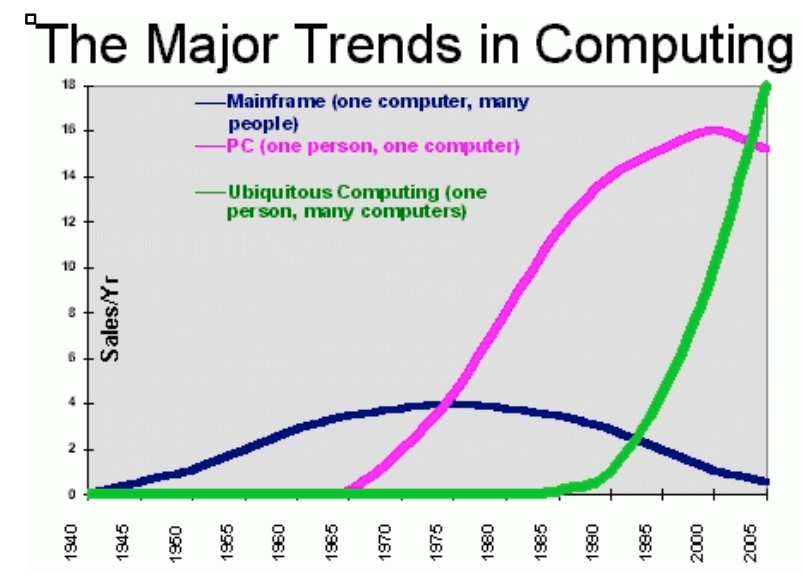

Figure 1. Slide from Weiser's presentation at Nomadic '96.

In a 'canonical' reading of this paper, it seems that future societal situations amenable to technological design and intervention were in PARC's case workplaces, which were seen as not being 'calm' enough due to the forecast massive increase in diverse technologies in the future of those workplaces [47] (see Figure 1, drawn from Weiser's presentation at Nomadic '96 conference [46]). Similarly, PARC's ubicomp envisioning drew on existing technology as a resource for extrapolation, such as the emergence of wireless networks, and the increased portability and miniaturisation of computational technology (e.g., early laptops). In addition to communicating their envisioning via publication, PARC also practically explored the envisioning through physical implementation via different scales of computational device $[43,44]$. This was a key component of the envisioning: testing out and experimenting withalbeit on a small scale — potential future technology designs and their implications for both technology and society.

One of the key methods Weiser employed for communicating this ubicomp envisioning in the SciAm article was a fictional, future scenario-the "Sal scenario". This describes a world in which 'Sal' lives, envisioning technology 
ideas through a science fiction narrative set in this future environment:

Sal awakens: she smells coffee. A few minutes ago her alarm clock, alerted by her restless rolling before waking, had quietly asked "coffee?", and she had mumbled "yes." "Yes" and "no" are the only words it knows.

Sal looks out her windows at her neighborhood. Sunlight and a fence are visible through one, but through others she sees electronic trails that have been kept for her of neighbors coming and going during the early morning. Privacy conventions and practical data rates prevent displaying video footage, but time markers and electronic tracks on the neighborhood map let Sal feel cozy in her street $[\ldots]$ [43]

The importance of fictions such as these is reflected in the longevity of impact of this particular scenario from the SciAm piece in orienting subsequent work. Mixing fiction with extrapolations, and notions of forecast and trajectory, offered a highly attractive and motivating envisioning; one which still fundamentally animates the field today. It is worth noting at this point here that I am less concerned with contributing a perspective on 'what was really meant', and instead exploring the ways in which this envisioning was subject to interpretation (and disputes about that interpretation), operationalised and, largely, concretised or frozen in subsequent work (cf. [4]). There appear to be some potential ways of making sense of this envisioning: as a forecast of future environments, as projection from existing technologies, and as a fictive scenario-based depiction of a socio-technical environment.

\section{Using ubicomp's envisioning}

Perhaps the most interesting aspect about this canonical perspective on ubicomp's envisioning is the way in which it has been interpreted and used.

Satyanarayanan's widely cited 2001 paper on ubicomp's "vision and challenges" envisions an "impressionistic picture of the road ahead" for pervasive and ubiquitous computing in order to determine a series of technology capabilities that researchers need to investigate in order to achieve the original envisioning. These areas include perennial ubicomp interests of context awareness, determining user intent, perceptual invisibility, mobile networking, location tracking, privacy issues, energy management and software adaptation (to name a few) [33]. Intriguingly, Satyanarayanan animates these research directions with future fictional scenarios, clearly inspired by the SciAm article, which "appear feasible in just a few years". In this way, these apparently fictional scenarios have been interpreted as a kind of forecast about what is technologically possible, thus supporting the areas in which research should be directed in order to adapt to this forecast feasibility.

A similar article (also widely cited) from Abowd \& Mynatt in 2000 reinforces ubicomp's future technological "trajectory" through a historical exploration of the 'story so far' and corresponding extrapolation to the "remaining challenges" [2]. Reflecting the societal aspect of ubicomp's envisioning, they suggest that the envisioning "offers the possibility of a killer existence". Accordingly, relevant technical areas are picked out from Weiser's envisioning, specifically: a focus on supporting everyday interactions, context awareness, capture of everyday experience, and 'natural' interfaces. In describing these, Abowd \& Mynatt explicitly refer to the need for extrapolative qualities of envisioning: "[d]esigning ubiquitous computing applications requires designers to project into the future how users will employ these new technologies". This, they argue is not a "new problem" but is "exacerbated" by designing for ubicomp futures.

Several years later, Adam Greenfield's 2006 book Everyware [17] provides a popular and accessible account of ubiquitous computing's history, current developments and future direction. In Everyware, Greenfield (self-described as a "critical futurist") discusses significant technological developments in ubiquitous computing, and describes the ways in which we must prepare ourselves for what these ubiquitous computing environments have in store for us as they emerge and impinge increasingly on our everyday lives. At the core of Greenfield's book is a series of ethical guidelines which he suggests will become central as ubiquitous computing develops.

It is my contention that in many ways, Everyware is quite revealing for the ubiquitous computing research programme. The field's presentation in the book relies heavily on envisioning work, and, like the papers discussed above, remains strongly oriented by the Weiser envisioning explored above. Everyware is directed by a chronological raft of developments in the field since the $90 \mathrm{~s}$, and is significantly oriented by subsequent uses and interpretations of the ubicomp envisioning. The book reflects this programme in the mirror of a mainstream, popular account; through this it enables our research community to explore how the field appears 'from the outside'.

In advancing his argument in Everyware, Greenfield summarises the progress of the ubicomp programme, exploring the trajectory of PARC's early work. Using this background milieu of existing and developing socio-technical infrastructures and innovations, drawing upon developments in diverse technologies such as mobile phones and other portable computation devices, RFID sensor networks, information appliances, and so on, Greenfield envisions a future world by extrapolating trends of miniaturisation, reduced cost of computation, and increasing storage space, in order to justify the development of ethical principles. These principles are used to forecast important future issues and challenges. The principles include prioritising user safety through 'default to harmless', promoting privacy and social agility (e.g., the importance of self-disclosing systems that offer fine-grained control for a users selfpresentation or "face"), the support for deniability and opting-out within our ubicomp systems, and the importance in conservation of users' time. 
Using Everyware lets us reflect upon the ways in which Weiser's envisioning has typically been used within the ubicomp programme. In doing this, the description of envisioning provided in the introduction can be refined.

Firstly, ubicomp's envisioning employs the observation that a raft of 'enabling' technologies already exist (e.g., for Greenfield these include address space via IPv6, QR codes, WiMax, ultra-wideband Bluetooth, etc.); it follows that this is the 'natural' environment in which ubiquitous computing-like technologies can and will flourish. Secondly, there is a projection from raw technology capabilities. This is the assertion that we can project future capabilities of essential enabling technologies. For instance, relying on future increases in storage space (e.g., hard disk size, solid state device size and volatile RAM), and, via Moore's Law, processor power and miniaturisation. Thirdly, there is projection from existing socio-technical systems. This is similar to the previous characteristic, however involves extrapolating from existing socio-technical systems, such as the use of RFID infrastructures as an embedded, de rigueur part of retail shopping. In the case of RFID, for instance, Greenfield suggests the viability of increased proliferation of ubiquitous sensing infrastructures in general. Fourthly, developing the previous point, these accounts have a reliance on proliferation. Cheaper, more powerful integrated chips and storage systems will suggest the possibility of embedding computing power in unlikely places, potentially everywhere. These apparently selfevident possibilities will appear attractive to various stakeholders in public and private sectors.

What is interesting about these various uses of the original ubicomp envisioning is their ahistoricality. Firstly they tend to ignore relationships to previous envisionings. For instance, the very technologies ubiquitous computing reacted against-VR in particular - also had an extensive relationship with development and communication of various envisionings, including significant forecasts, extrapolations and fictions. Secondly, uses of ubicomp's envisioning tend to make implicit assumptions regarding the purposes of, the audience of and context in which the envisioning was created. The next sections will address this ahistoricality by reviewing just these two underplayed aspects.

\section{ENVISIONING VIRTUAL REALITY}

The development of VR, particularly during the early 1980 s to 1990 s, took place in an environment in which future-oriented design thinking played a considerable role. Commonplace within this environment were the extrapolations, forecasts and fictions of VR envisionings - the characteristics of which can provide a useful comparative context to understand ubicomp envisionings and their various interpretations and uses.

VR envisionings concerned themselves with a societychanging vision of future in which VR technologies would make a large contribution to everyday life. In an analysis of the developments in virtual reality during the years 19841992 [11], Chesher highlights in the discourse surrounding the raw technologies being developed for VR systems, the visions, forecasts and predictions for what nascent VR technologies would do for society and how they would impact and change the way digital media was experienced.

Early virtual reality pioneers situated the developments of the late 1980s within the context of a 'grand historical narrative', retrospectively constructing a timeline in which previous VR-like systems, such as the 1960s Sensorama were cast as the progenitors of existing ones:

In terms of VR history, putting my hands and head into Sensorama was a bit like looking up the Wright Brothers and taking their original prototype out for a spin. (Rheingold, quoted in [11])

Chesher describes how a central component of this narrative construction involved situating recent developments in VR (as of the early 1990s) within a larger context, and so using these as multiple 'data points' in order to project virtual reality technology's future significance:

My idea of virtual reality is that, in the long run, it's going to shut down television. (Lanier, quoted in [11].)

In essence, a paradigm shift expands the potential of an entire discipline... Computers are not just symbol processors, they are reality generators. (Bricken, quoted in [11])

Chesher illustrates the widespread tendency across research cultures as well as the media to envision VR as a significant 'paradigm shift' (just as ubicomp has frequently been characterised as, e.g., $[16,2,1])$, with its forecast future as a game-changing technology comparable to what had been experienced with the advent of previous revolutionary technologies such as radio, telephony, the moving image, television, and the computer itself, which had, at their own emergence, been treated in a similar manner (as have now forgotten technologies) [36].

Discourse in VR has been occupied with the extensive use of analogies relating the "colonization of cyberspace" with a "new frontier", drawing heavily upon the mythology and lore of the American Old West in the late 19th century [11]. Again, echoes in ubicomp are forthcoming: Satyanarayanan's envisioning paper describes pervasive computing as being similar to "the Frontier of the American West in the early 19th century", i.e., "a rich open space where the rules have yet to be written and the borders yet to be drawn" [33].

Like the sense of 'trajectory' for ubicomp, the prevalent discourse regarding virtual reality was a somewhat utopian, teleological sense of an emerging technology; for some VR was seen as the necessary result of a historical technological narrative encompassing the history of telecommunications equipment and the computer's development. The fulfilling of virtual reality technology's potential was always 'just around the corner' according to this narrative. Notably, Weiser's SciAm article lightly employed this sense of trajectory, describing the then-current technologies as "analogous to the period when scribes had to know as much about making ink or baking clay as they did about writing". 
More strongly teleological sentiments have also been expressed arguing that, in pursuing the development of ubicomp technologies, the "promise of ubiquitous computing will eventually be fulfilled" [29]. Other examples are forthcoming (e.g., [33, 2, 29, 17]; see [4]).

Fictional influence has also been key for VR, with popular science fiction books such as Gibson's Neuromancer providing a background for and at times directly influencing technology development. Gibson depicts a worldwide "consensual hallucination" of cyberspace-an immersive dataspace in which users conduct their everyday lives in terms of both business and leisure. Bleecker reports that Neuromancer was considered a kind of "shared technical manual" for VR pioneers at the Human Interface Technology Lab, Washington [7]. Fictional sources such as these were often co-opted by the envisioning process as forecast future scenarios, perhaps at times ignoring the sense of irony (or fiction) with which they were created:

While Gibson's vision is beyond the reach of today's technology, it is nonetheless, today, possible to achieve many of the effects alluded. A number of companies and organisations are actively developing the essential elements of a cyberspace deck... (Walser, quoted in [11])

Ultimately, increasing embarrassment over the hyperbole and apparent inaccuracies of VR envisionings set in, particularly as applications of VR turned out quite differently to forecasts developed via the envisioning process. VR technology has since not offered a "new frontier" or "shut down" television or other existing media, nor even become a ubiquitous method for running our everyday affairs. However, immersive VR (i.e., involving specialist input and output devices such as head-mounted displays, wearable interfaces, and body tracking) has found limited specialist uses, such as in interactive installations, military training simulators, or treating phobias (e.g., [28]). Nonimmersive VR itself has become a de-facto environment for video games. But this is, deeply, radically different to the envisionings of the late 1980s and early 1990s. From the perspective of how these envisionings appear to have been interpreted, i.e., often as apparent forecasts about the future, they were manifestly 'wrong'.

One of the key points of comparison with ubiquitous computing's envisionings is the mixture and potential conflation of forecasts, extrapolations and fictions. VR provides us with another demonstration of an envisioning that mixes these various components. I argue that a key 'stimulant' within envisionings' mix of these components is the use of fiction; this is witnessable in the impact and longevity of the Sal scenario (e.g., $[33,19])$. The tendency not to unpick these components from one another has led to a propensity to conflate fiction, extrapolation and forecast.

This orientation can be found in the reception of much ubiquitous computing work inspired by PARC onwards. At times there is a curious relationship between academic papers, films and promotional videos. Although it seems that the greatest blurring or conflation of forecast, extrapo- lation and fiction may be found in the popular media (since journalistic accounts typically rely on this blurring in order to perform the translation of research into journalism), there can be and has been a significant relationship with research work [15]. For instance, as part of the making of the sci-fi film Minority Report, the producers "convened a three-day conference about what life will be like in the year 2054" [12], inviting, amongst others, technologists and writers. The production of the film was also notable in that it purposefully employed members of MIT Media Lab-a prolific contributor to ubicomp and HCI communitiesamongst its technical advisory team, including John Underkoffler. Underkoffler would go on to produce a 'Minority Report' gestural interface, which he demoed at Technology, Entertainment, Design (TED) 2010 conference [42]. It is important to see this within the context of a wider historical trend, i.e., that of substantial feedback between the science fiction imagination and technological development $[15,22]$. This tangling of the boundaries between pragmatic, concrete workaday technology research and projected futures of that technology within society seems to be characteristic of communities which employ envisioning.

In closing this exploration of $\mathrm{VR}$, it is instructive to reflect upon ubiquitous computing's initial explicit framing as a response to virtual reality [43] - i.e., instead of technology being used to draw users into a generated reality, technology is instead seen as being designed to fit in with the reality of the everyday world. In this sense ubicomp is the inverse of virtual reality ideals - immersion in the world with technology rather than immersion in a virtual world that eschews the physical world. However, in spite of this, and as I have shown in this section, the similarities between the details of ubicomp and VR envisionings are striking: the use of similar appeals to Old West 'frontier' metaphors in the ubiquitous and pervasive computing literatures; discourses of 'paradigm shifts'; extrapolations from existing technology capabilities; a teleological sense of inevitability derived from the certainties of technological progress; the influence and deployment of fictions as part of the technology culture; and, crucially, the mixing and conflation of these extrapolations, forecasts and fictions.

It is worth noting before moving on that VR has not been the only influential envisioning in computer science. In some sense computer science gains some of its earliest orientations from envisionings: for instance, John von Neumann, a significant contributor to the foundations of computer science, claimed that "the ever-accelerating progress of technology... gives the appearance of approaching some essential singularity in the history of the race beyond which human affairs, as we know them, could not continue" [38, p. 386]. Beyond this are numerous other examples: the envisioning of future workplaces deployed notions of the 'paperless office' which repeatedly fell out of step with real world work practices [34]; video conferencing's envisioned ubiquity within business domains never matched reality, and has directed focus away from its underexamined prevalence within domestic environments [23]; 
and the supposed dominance of 'information appliances' [3] has been repackaged as another envisioning, the 'internet of things'.

Each of the examples reviewed in this section can offer us lessons for ubicomp's uses of envisioning, particularly that envisionings can be highly fragile things when deployed without reference. Thus I argue that we can start understanding ubiquitous computing's envisionings through studying other envisionings.

\section{INTERPRETING UBICOMP'S ENVISIONING}

The introduction weighed the importance of how envisionings come to be interpreted over the original intentions of envisionings' creators. Here I unpack this notion in more detail, focussing on the role of forecasts in particular, in addition to discussing other critiques of ubicomp's original envisioning. Finally, I present some comments on 'reading' envisionings which attempts to throw these other interpretations into relief.

\section{Envisionings and forecasts}

A recurrent way of interpreting the canonical ubicomp envisioning has been in terms of how its details match up with the present day. This perspective often trades on reading the envisioning as a forecast that can be evaluated for how accurately it matches with present trajectories, based on extrapolations from current technological capabilities. This section addresses forecasting's accountable character, and the role of uncertainty in forecasting.

In many situations, forecasting is a morally accountable activity. Correspondingly, envisionings read as forecasts are often seen in this way; should they fail to provide an accurate reading as forecasts, they can be interpreted as a breach of the moral order, e.g., a 'broken promise' [18]. In this view of envisionings as accountable, it becomes possible to begin to see dissonances between the early ubiquitous computing envisioning and the reality of subsequent technology developments [3]. For instance, questions have been raised about early technology demonstrators; as Lueg notes: "[a] decade later we know that despite offering certain benefit, something like the active badge location system did not become standard office equipment $[\ldots]$ it is reasonable to assume that it is not technical problems that have prevented such systems from becoming standard office equipment" [26]. Bell and Dourish report directly on this issue, suggesting that 'ubiquitous computing' has arrived into our everyday lives, but we have not recognised it due to it being different to the canonical envisioning [4]. In this way the envisioning has often been seen by the ubicomp and HCI community as something that can legitimately be judged in terms of accuracy (i.e., as a forecast).

As shown earlier, particularly with Greenfield's use of ubicomp envisionings, one of the critical foundations that is often employed is Moore's Law. Moore's Law is routinely appealed to implicitly and explicitly in order to buttress ubicomp envisioning's extrapolations from raw technology capabilities into future ubicomp environments. Moore's Law began as a simple observation on the relationship between the number of transistors manufacturers could fit onto integrated chips, and time. This relationship seemingly allows us to 'forecast' the progress of computation, such as how much computational power we can expect in the future. (There are congruities with other aspects of technology developments, such as increased storage capacities, bandwidths, and so on.) But Moore's Law is not a natural law; instead, it is a self-referential forecast about the future of a socio-technical environment that involves complex and unpredictable feedbacks. Manufacturers of integrated chips themselves use projections of Moore's Law as production targets for the expected number of transistors they will try to fit on a chip [10]. In this way the socio-technical forecasts of Moore's Law become folded into readings of ubiquitous computing's envisionings, and so bundled in with their accountable character.

When interpreting envisionings as forecasts we can also hit the problems of uncertainty, and miss important unenvisioned developments. Taleb's work on uncertainty is instructive here [39]. Specifically, he argues that forecasts are vulnerable to so-called 'Black Swan events'. These events are rare, unenvisioned and extremely hard to predict, but carry a large, unforeseen impact. The emergence of the web is a prime example, being originally developed for "no loftier reason than to help [Berners-Lee and research scientists at CERN] remember the connections among [...] various people, computers, and projects" [6, p. 4].

It is these unforeseen technological Black Swans which envisionings interpreted as forecasts can miss. Taleb suggests that uncertainty generally and Black Swan events specifically are often 'explained away' (such as suggesting Vannevar Bush's famous 'As we may think' forecast the web), given a trajectory, and narratised post hoc such that they appear within a predictable, linear narrative, often coupled with the suggestion that more could have been done or known about at the time to successfully predict the event. Using work by Tversky and Kahnemann, Taleb argues that we consistently and repeatedly failed to both foresee the number of Black Swan events and anticipate the extent of their impact; furthermore, we 'forget' that we have done so, hence leading to a sense of ahistoricality (e.g., not comparing ubicomp envisionings to VR envisionings in spite of Weiser's treatment of them).

\section{'What was really meant'}

Largely this paper has examined what I see as the most widespread, canonical ways of interpreting Weiser's envisioning, but it would be unfair to present the interpretation of it as uncontested. For example, Rogers expresses concern with the SciAm envisioning of "calm computing" [47]. She argues that the way it has practically and conceptually oriented ubicomp research has necessarily excluded what more "engaging user experiences" [32]. However, this critique does not question the ways in which the SciAm piece has been deployed, its precedents, what its original audience might have been, or context of publication. 
Similarly, when questions are raised over interpretation of the envisioning, we often see this discussion being framed in terms of 'what was really meant'. For instance, Weiser's envisioning made heavy use of the notion of 'invisibility' [45], which has routinely been interpreted as a perceptual invisibility rather than a sense of the everyday and 'unremarkable' [41].

In turn, Bell \& Dourish argue that Weiser's SciAm article has resulted in ubicomp's community being concerned with "yesterday's tomorrows", i.e., implementing the envisionings of the past in order to decide how to build things of the future [4]. Instead they suggest that "ubiquitous computing is already here; it simply has not taken the form that we originally envisaged and continue to conjure in our visions of tomorrow". Ubicomp has missed the 'messy' reality of these existing real-world ubicomp environments. In this way, Bell \& Dourish take the impact of the envisioning to task, but do not unpack the envisioning process itself.

\section{Reading envisionings}

Instead, I would like to contrast these with an alternative reading of the envisioning. Firstly it is possible to unpack the extrapolations, the forecasts and the fictions from one another. The scenario may be read as a fiction, coupled with the intrinsic features that fictions are written withe.g., that they are not morally accountable forecasts.

Instead the SciAm article is illustrative of the principles and values inherent in the new technology, but at the same time represents a public maintenance of CSL's (and therefore PARC's) relationship to its parent company, Xerox. During the emergence of ubiquitous computing's envisioning it is of note that in 1994 Xerox was undergoing a transformation from being 'the photocopier company' to, as its rebranding represented, "The Document Company" [13], i.e., a company providing a complete document service rather than just products. In this way, with Xerox themselves forming a significant part of the intended audience for Weiser's article, the envisioning was no doubt sensitive of the shift and indeed oriented towards it. This reading is supported by the envisioning being presented to a popular science audience that could include Xerox management, rather than an audience of HCI researchers, who would subsequently cite this article so heavily. Indeed, many envisionings are 'for' sponsors or funding bodies in shaping such a relationship [25]. In comparison, it is notable that a later article explicitly for a computer science audience (Comm. ACM) would emphasise 'computer science issues' [44], rather than elaborating on a future world via fictions and forecasts [43].

\section{DISCUSSION}

This paper has exhibited a number of recurrent characteristics of envisioning that, while prominent in the ubicomp programme, are also present in other envisionings. In essence, envisioning is about how existing socio-technical environments will develop, what new ones will emerge, enabling us to reason about what to design, what not to design and how to design. In doing this, envisioning uses elements of fiction, extrapolation and forecasting, mixing its relationship with science fiction, scenarios, and popular culture, with apparent technological facts such as the existence of 'enabling' technologies, Moore's Law, and technology proliferation trends.

This paper has for the most part been concerned with trying to disentangle ways in which these envisionings can be 'read' and interpreted. Envisionings can be problematic to read due to issues of conflation, context and audience. In this way I have sought to problematise envisioning, its histories and its interpretation. This discussion seeks to set these difficulties alongside ways in which envisioning can also be useful, and often vital. In order to be aware of the benefits as well as the dangers of envisioning, we need to foreground many of its implicit aspects. Primarily, I feel that we need to learn how to read and apply envisionings in order to understand the work they are doing for us in progressing the field.

In this way, this discussion attempts to raise a number of issues that, I argue, should either become part of discourse in HCI or gain greater visibility: the role of teleologies and narratives in our research programmes; technology forecasting and the role of 'the present'; ways of disentangling fictions, forecasts and extrapolations; and the pros and cons of practically engaging with envisionings in research programmes.

\section{Teleology, narrative and trajectory}

A key feature of envisioning is the use of technological trajectories and narratives about an imagined technology's precursors and its extrapolated future. At times this assumes a teleological character, i.e., that these narratives reach a definite endpoint. von Neumann's remarks (i.e., [38, p. 386]) highlight how these teleological notions have cropped up from time to time in computer science culture since its beginnings. Often this perspective is manifest through arguments of technological determinism regarding how the relationship between technology and society is understood. The culture of the ubicomp programme that is represented in Everyware remains determinist:

And although I would prefer to resist determinism in any of its forms, above all the technological, it's hard to argue with in this instance $[. .$.$] everything digital can by its very$ nature be yoked together, and will be. [17, p. 97]

This reflects a wider malaise. Taylor and Harper [37] suggest that in spite of determinism within HCI becoming increasingly criticised, "deterministic talk continues to be conspicuous in the rhetoric surrounding wireless, mobile technologies". Within ubiquitous computing envisioning, "overly deterministic visions of technology have misjudged, not only the downturns in economic cycles, but also how we, as active members of society, come to shape technology for ourselves".

This calls for greater reflection upon the way we make narratives of past, present and future technologies. I argue that we need to start questioning our application of envisionings as frameworks, and examine whether envisionings 
are grossly simplifying our understandings of the role of technology in society through narratives and trajectories that necessarily exclude elements that don't quite fit the story, i.e., confirmation bias.

\section{'Future' is the present (and sometimes the past)}

Envisionings can at best only ever be 'about' the present. Philips" "Vision of the Future" project [24] of 1995 is a case in point; "[o]ur concern was not only in technologies that would be mature by the year 2005, but also in technologies which would begin to emerge at that time". Through this Lambourne et al. raise a set of future concerns for 10 years hence (i.e., 2005) such as design for sociability, exploration, connectivity, ethics, and subjectivity. These future issues are framed entirely within the concerns of technology in 1995. For instance, there are references to forecast exploration of "a new world" or "that of "cyberspace', the "virtual' world", strongly reflecting the discourse at the time that surrounded virtual reality. We also saw how similar 'future' challenges were presented in Everyware (albeit minus any VR references).

In reality envisionings only ever (and can only ever) reflect the concerns of the time, and should be read as such; they are a discursive or scenic feature of the design spaces we work in [35]. In some cases, envisionings tie us to the concerns of the past at the expense of explicitly tackling the issues of the present ("yesterday's tomorrows" [4]). This means envisioning can only create principles about the present (e.g., Philip's or Greenfield's), not predictions of the future. Twisting envisionings in this way means embracing the production of principles for design rather than designs from forecasts of use or situation. Redström [31], quoting Akrich, suggests that "[a] large part of the work of innovators is that of 'inscribing' this vision of (or prediction about) the world in the technical content of the new object". Thus, principles expressed in designs are questions for future use. These principles are explicitly tied to the context in which they are produced and not contingent upon a forecast future. They promote the acceptance of uncertainty as a valid (and animating) feature of research work, and trial and error as a way of continually recalibrating and refining those principles.

\section{Disentangling fiction from forecasting \& extrapolation}

I have shown how extrapolations and forecasts are often freely mixed with fictional aspects in envisionings. For example, if we read fictional scenario components of envisionings more as forecasts or extrapolations of the present, we will no doubt find that they employ significant assumptions about the progress of technology in order to animate them, in spite of those assumptions glossing huge and nontrivial technical problems (e.g., context-awareness, as per Weiser's scenario) [26]. As outlined in this paper, there are some fragilities to forecasting, such that it is important to consider what it means to interpret an envisioning as forecast rather than fiction. As such I would argue that we need to disentangle fiction from its more fragile forecasting and extrapolative aspects.
This might mean, meaning that envisioning should more often be treated explicitly fiction. Fiction is a powerful, creative and playful way to reason about what we are to do in the future. Fiction guards against the teleological tendencies of forecasting, against explaining away 'bad' predictions and lauding 'accurate' ones. As a creative endeavour, fiction opens up possibilities that forecast tend to shut down. Fiction transforms the assumptions of envisioning that forecasting employs - that there are 'enabling' technologies for the future, that we can project from existing capabilities, that we can rely on proliferation of technology, or that we can imagine future societal situations-and instead uses those assumptions to drive design thinking about the present.

Design fiction [7] and scenarios can offer an explicitly fictional approach that engages with futures, yet challenges envisioning's assumptions. Design fiction in particular "let[s] go of convention and expectations about how the future looks, the direction to which progress is meant to go, constructively imagining that there are multiple possible futures rather than one future that goes in one direction (up and to the right), or one future, evenly distributed" [7]. Blythe \& Wright, in turn, detail an approach in which fiction becomes a resource for user-centred design [8].

\section{Focus versus diversity}

Ubicomp's envisioning has focussed research endeavours to explore a range of areas and problems: Rogers suggests "[m]ost prominent themes are context-aware computing, ambient / ubiquitous intelligence and recording / tracking and monitoring" [32]. Satyanarayanan [33] and Abowd \& Mynatt [2] come to similar conclusions, and these themes can be accounted for quite directly as interpretations of Weiser's original envisioning. In articulating this, envisionings can create remarkable motivation, foster community, and assist in attracting funding for tackling a particular set of problems and areas that serve the envisioning. Ubicomp in many ways has quite successfully unpacked and explored a range of significant technical and social challenges oriented via these themes.

Such focus can be powerful for all the above reasons; it enables resources to be focussed in directed programmes to structure how specific problems are tackled. However, there are potentially negative aspects. Envisionings create a tendency to homogenise and corral technology research work towards a particular interpretation or set of canonical forms of the envisioning. Interpretations of PARC envisionings, for instance, favoured 'calm' solutions, perhaps to the detriment of 'engaging' solutions [32], and perceptual invisibility instead of understanding ubicomp invisibility as 'unremarkable' in use [41]. This envisioning-oriented subdivision at worst can lead to a form of research siloing or 'Balkanisation'.

Using envisioning to focus and plan for action cannot come at the expense of more diverse programmes that do not rely upon envisionings. We must also develop research that sits purposefully outside of the envisioning, as part of recogni- 
tion of inherent uncertainty. Diversity of approach and dynamism strengthen our ability to handle Black Swan technologies. This goes some way to explaining why it is that ubicomp's programme, in being focussed on, oriented by and interpreting a particular envisioning, has tended to miss what is happening in the present that could be considered 'ubicomp' [4]. Large influences can come from outside the envisioned setting; for instance, the natural world (e.g., climate change) may impinge upon technology development (cf. the arguably late emergence of interest in sustainable computing, ICT4D, etc.).

Fictional and scenario design approaches that challenge envisioning's assumptions are often situated in terms of dichotomies and binaries, such as utopian versus dystopian interpretations of ubicomp envisioning (e.g., [27]). Population-based approaches offer one technique for practically addressing this in scenario design. Davidoff et al. [14] suggest one such population-based participatory approach for design in an uncertain environment, involving sketching multiple alternative fictional scenarios and subsequently exploring them, discarding irrelevant designs and retaining the most successful. Comparing the above approaches, it is clear that using diversity as a key guiding principle requires greater resources, however.

\section{CONCLUSION}

This paper has focussed on envisionings, how they feature specifically within the ubicomp programme, and HCI more broadly. In unpacking envisioning's role within early and more contemporary ubiquitous computing, I have sought to compare ubicomp with other envisionings, such as that of VR. This has been a way of reflecting on ubiquitous computing's culture and practices. Through considering the ways in which envisionings may be read, this paper has advocated the need for a gestalt shift. This shift primarily is about opening up a discussion in $\mathrm{HCI}$, and foregrounding the envisioning process; through an envisioning's creation to subsequent interpretation and reinterpretation.

This paper's main practical contribution to HCI is a set of key sensitising concepts for envisioning outlined in the discussion: the role of teleology; the role of the present and past in 'the future'; the entangling of fiction with forecasting and extrapolation; and trade-offs between focus and diversity. A series of questions can be developed from this:

- Who might be the intended audience(s) of the envisioning?

- What is the context in which the envisioning has been created?

- What happens if the envisioning is read in fictional ways? Or read as forecasts or extrapolations?

- What issues of the present is the envisioning concerned with and speaking to?

- What is the relationship of the envisioning to other, older envisionings? What can we learn from them?

- How is the envisioning using narratives, and what 'endpoints' or target situations are assumed?
- In using a particular envisioning, what pathways might we be shutting down as possibilities, which endpoints might be excluded, which present issues are excluded?

These questions can be integrated into design processes and techniques such as paper prototyping, participatory design, storyboarding, scenario design, brainstorming, and concept clustering. This could mean, say, explicitly creating multiple scenarios based on different envisionings, rather than working under the implicit assumption of only one particular envisioning. Or it could help identify which present socio-technical issues that, say, a paper prototyping process is actually speaking to. Integrating these questions within design methods could take a number of forms, ranging from a background mindfulness through to the questions forming an explicit stage in that design process.

\section{ACKNOWLEDGEMENTS}

This work is supported by Horizon Digital Economy Research, RCUK grant EP/G065802/1. Thanks to all those who have engaged in dialogue on this work (including reviewers); I only have space to highlight a few: Bob Anderson, Murray Goulden, Robert Dingwall and Dave Kirk.

\section{REFERENCES}

1. Abowd, G. Software engineering issues for ubiquitous computing. In Proc. of ICSE '99, pp. 75-84. ACM Press, 1999.

2. Abowd, G. \& Mynatt, E. Charting past, present, and future research in ubiquitous computing. ACM Transactions on Computer-Human Interaction. 7(1):29-58. ACM Press, 2000.

3. Barkhuus, L. \& Polichar, V. E. Empowerment through seamfulness: smart phones in everyday life. Personal and Ubiquitous Computing, 15(6):629-639. Springer, 2010.

4. Bell, G. \& Dourish, P. Yesterday's tomorrows: notes on ubiquitous computing's dominant vision. Personal Ubiquitous Computing, 11(2):133-143, 2007.

5. Begole, B. \& Masuoka, R. Search for Eden: Historic perspectives and current trends toward the ubiquitous computing vision of effortless living. Information Processing Society of Japan Magazine, 49(6):634-640, 2008.

6. Berners-Lee, T. Weaving the Web. HarperOne, 1999.

7. Bleecker, J. Design fiction: A short essay on design, science, fact and fiction. Near Future Laboratory, 2009.

8. Blythe, M. A. and Wright, P. C. Pastiche scenarios: Fiction as a resource for user centred design. Interacting with Computers, 18(5):1139-1164, Elsevier, 2006.

9. Brown, N., B. Rappert, and A. Webster (eds). Contested Futures: a sociology of prospective techno-science. Ashgate, 2000.

10. Ceruzzi, P. E. Moore's law and technological determinism: reflections on the history of technology. Technology and Culture, 46:584-93, 2005.

11. Chesher, C. Colonizing virtual reality construction of the discourse of virtual reality, 1984-1992. Cultronix, 1(1), 1994. 
12. Clarke, D. J. MIT grad directs Spielberg in the science of moviemaking, http://bit.ly/bTXbjR (verified 06/09/11)

13. Collins, G. "Xerox attempts a new beginning by making its name the last word in corporate rechristening". New York Times, August 4th, 1994, p. 17.

14. Davidoff, S., Lee, M. K., Dey, A. K. \& Zimmerman, J. Rapidly Exploring Application Design Through Speed Dating. In Proc. UbiComp, pp. 429-446. SpringerVerlag, 2007.

15. Dourish, P. \& Bell, G. "Resistance is Futile": Reading Science Fiction Alongside Ubiquitous Computing. To appear in Personal and Ubiquitous Computing.

16. Galloway, A. Understanding emergent technologies. Chapter 4, unpublished $\mathrm{PhD}$ thesis, Carleton University, Canada, 2008.

17. Greenfield, A. Everyware: the Dawning Age of Ubiquitous Computing. Peachpit Press, 2006.

18. Horner, D. S. Digital futures: promising ethics and the ethics of promising. ACM SIGCAS Computers and Society. 37(2):64-77. ACM Press, 2007.

19. Indulska, J. \& Robinson, R. Modelling Weiser's "Sal" scenario with CML. In Proc. PERCOM, pp. 1-6, IEEE Computer Society, 2009.

20. Johnson, B. D. Science Fiction Prototyping: Designing the Future with Science Fiction, Synthesis Lectures on Computer Science, 3(1), Morgan \& Claypool, April 2011

21. Kinsley, S. Anticipating ubiquitous computing: Logics to forecast technological futures. Geoforum, 42(2):231240, 2011.

22. Kirby, D. The Future is now: Diegetic Prototypes and the Role of Popular Films in Generating Real-World Technological Development. Social Studies of Science, 40(1):41-70, 2009.

23. Kirk, D. S., Sellen, A. \& Cao, X. Home video communication: mediating 'closeness'. In Proc. $C S C W$, pp. 135-144. ACM Press, 2010.

24. Lambourne, R., Feiz, K, \& Rigot, B. Social trends and product opportunities: Philips' Vision of the Future project. In Proc. CHI, pp. 494-501. ACM Press, 1997.

25. Lesser, L. I., Ebbeling, C. B., Goozner, M., Wypij, D. \& Ludwig, D. S. Relationship between Funding Source and Conclusion among Nutrition-Related Scientific Articles. PLoS Med, 4(1):e5, 2007.

26. Lueg, C. On the Gap between Vision and Feasibility. In Proc. Pervasive, pp. 45-57. Springer-Verlag, 2002.

27. Mancini, C., Rogers, Y., Bandara, A. K., Coe, T., Jedrzejczyk, L., Joinson, A. N., Price, B. A., Thomas, K., \& Nuseibeh, B. Contravision: exploring users' reactions to futuristic technology. In Proc. CHI, pp. 153162. ACM Press, 2010.

28. Moore, K., Wiederhold, B. K., Wiederhold, M. D., Riva, G. Panic and agoraphobia in a virtual world. $C y$ berpsychology \& Behavior, 5(3), pp. 197-202, 2002.

29. Newman, M. W., Ducheneaut, N., Edwards, W. K., Sedivy, J. Z., \& Smith, T. F. Supporting the unremarka- ble: experiences with the obje display mirror. Personal Ubiquitous Computing, 11(7):523-536, 2007.

30. Patel, D. \& Pearson, I. D. Hype and Reality in the Future Home. BT Technology Journal, 20(2), pp. 106-115, April 2002.

31. Redström, J. Towards user design? on the shift from object to user as the subject of design. Design Studies, 27(2):123-139, 2006.

32. Rogers, Y. Moving on from Weiser's vision of calm computing: engaging ubicomp experiences. In Proc. Ubicomp, pp. 404-421. Springer, 2006.

33. Satyanarayanan, M. Pervasive computing: vision and challenges, Personal Communications, IEEE, 8(4):1017, 2001.

34. Sellen, A. J. \& Harper, R. H. The Myth of the Paperless Office. MIT Press, 2001.

35. Sharrock, W. and Anderson, R. The user as a scenic feature of the design space. Design Studies, 15(1):5-18, 1994.

36. Sturken, M., Douglas, T., \& Ball-Rokeach, S. (eds.) Technological visions: the hopes and fears that shape new technologies. Temple University Press, Philadelphia, 2004.

37. Taylor, A. S. and Harper, R. Age-old practices in the 'new world': a study of gift-giving between teenage mobile phone users. In Proc. CHI, pp. 439-446, ACM Press, 2002.

38. Teuscher, C. (ed) Alan Turing: life and legacy of a great thinker. Springer, 2003.

39. Taleb, N. N. The Black Swan: The Impact of the Highly Improbable. Random House, 2007.

40. Taleb, N. N. The Fourth Quadrant: A map of the limits of statistics. Edge Foundation, 2008. http://bit.ly/OdEMS (verified 21/09/11).

41. Tolmie, P., Pycock, J., Diggins, T., MacLean, A. \& Karsenty, A. Unremarkable computing. In Proc. CHI, pp. 399-406, ACM Press, 2002.

42. Underkoffler, J. Talk given at TED 2010, http://bit.ly/aaIaBe (verified 06/09/11)

43. Weiser, M. The computer for the twenty-first century. Scientific American, pp. 94-104, Sept. 1991.

44. Weiser, M. Some computer science issues in ubiquitous computing. Communications of the ACM, 36(7):75-84, 1993.

45. Weiser, M. The world is not a desktop. interactions, 1(1), pp. 7-8, Jan. 1994.

46. Weiser, M. Nomadic Issues in Ubiquitous Computing, talk given at Nomadic '96, 1996. http://bit.ly/r27EKM (verified 06/09/11).

47. Weiser, M. \& Seely Brown, J. The Coming Age of Calm Technology. In Beyond Calculation: The Next Fifty Years of Computing, Denning, P. J. \& Metcalfe, R. M. (eds.), Springer-Verlag, 1997. 